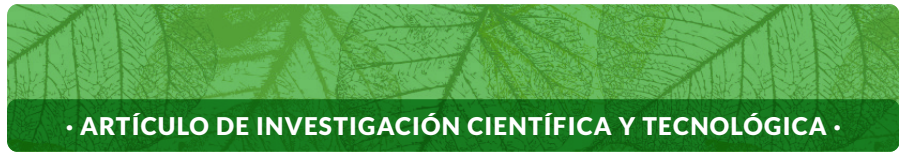

Vol. 19 No 2 • Julio - Diciembre 2021 • ISSN - 1692-3561 • ISSN-e 1909-9959 • https://doi.org/10.18684/bsaa.v19.n2.2021.1363

\title{
Aceite esencial de orégano (Lippia origanoides) mejora parámetros productivos y metabólitos sanguíneos en lechones*
}

\section{Oregano essential oil (Lippia origanoids) improves productive parameters and blood metabolites in piglets}

\author{
MAYA-ORTEGA, CARLOS-ABELํㅜ ÁNGEL-ISAZA, JAIME-ANDRÉS; \\ MARTÍNEZ-MORALES, BLANCA-CECILIA³;PARRA-SUESCÚN, JAIME-EDUARDO ${ }^{4}$
}

Historial del Artículo

Recibido para evaluación: 16 de Junio 2020.

Aprobado para publicación: 22 de Enero 2021.

* $\quad$ Proyecto de origen: "Genotopificación de la microbiota intestinal en cerdos recién destetados luego de la adición de diferentes compuestos antimicrobianos en el alimento". Financiación: Universidad Nacional de Colombia sede Medellín y PROMITEC Santander SAS. Culminación: octubre 22 de 2020

1 Universidad Nacional de Colombia, Sede Medellín, Facultad de Ciencias Agrarias, Departamento de Producción Animal, Grupo de investigación BIOGEM. Msc Zootecnia. Medellín, Colombia. https://orcid.org/0000-0002-5094-7716

2 PROMITEC Santanter S.A.S, Grupo de investigación, desarrollo e innovacion Promitec Santander Grupo. MVZ, MsC. Santander, Colombia. https://orcid.org/0000-0003-1612-6938

3 PROMITEC Santanter S.A.S, Grupo de investigación, desarrollo e innovacion Promitec Santander Grupo. Quimica. Santander, Colombia. https://orcid.org/0000-0002-8417-8687

4 Universidad Nacional de Colombia, Sede Medellín, Facultad de Ciencias Agrarias, Profesor Departamento de Producción Animal, Grupo de investigación BIOGEM. Zoot, MsC, Ph.D. Ciencias agrarias. Medellín, Colombia. https://orcid.org/0000-0003-4772-1326 


\section{RESUMEN}

Los antibióticos han sido utilizados en la alimentación animal con el fin de garantizar una producción más eficiente; sin embargo, la aparición de bacterias resistentes a los antibióticos ha causado preocupación tanto a consumidores como científicos. El objetivo fue evaluar el efecto de la suplementación con Lippia origanoides sobre el rendimiento productivo y la concentración de metabolitos sanguíneos en lechones destetados a los 21 días. Se utilizaron 72 lechones destetados el día 21 y aleatorizados a 3 dietas: (D1) alimento base, (D2) alimento base + Bacitracina de zinc (350 ppm) y (D3) alimento base + Lippia origanoides (150 ppm). Los días 15 y 30 del periodo experimental se evaluaron parámetros zootécnicos y variables metabólicas a nivel sérico. Los animales que consumieron la dieta D3 presentaron mayor conversión alimenticia y ganancia de peso, además de presentar concentraciones mayores $(p<0,05)$ de fosfatasa alcalina, calcio y fosforo, mientras que las concentraciones de creatinina, alanina aminotransferasa, glucosa, triglicéridos y colesterol se encontraron significativamente $(p<0,05)$ inferiores en comparación con los animales que consumieron (D1) y (D2). El uso de Lippia origanoides en la dieta de lechones constituye una alternativa al uso de antibióticos, pues tiene efectos favorables sobre la bioquímica sanguínea y el rendimiento productivo.

\section{ABSTRACT}

Antibiotics have been used in animal feed in order to ensure more efficient production, however, the appearance of antibiotic resistant bacteria has caused concern in both consumers and scientific. The aim was to evaluate the effect of Lippia origanoides supplementation on the productive performance and the concentration of blood metabolites in piglets weaned at 21 days. 72 piglets weaned on day 21 were used and randomized to 3 diets: (D1) basic feed, (D2) basic feed + zinc bacitracin (350 ppm) and (D3) basic feed + Lippia origanoides (150 ppm). On days 15 and 30 of the experimental period, performance was calculated and metabolic variables were evaluated at serum level. The animals that consumed the $D 3$ diet presented higher feed conversion rate and body weight gain, in addition to presenting higher concentrations ( $p<0,05$ ) of alkaline phosphatase, calcium and phosphorus, while the concentrations of creatinine, alanine aminotransferase, glucose, triglycerides and cholesterol were found significantly $(p<0,05)$ lower compared to animals that consumed (D1) and (D2). The use of Lippia Origanoides in the piglet diet is an alternative to the use of antibiotics, since it has favorable effects on blood biochemistry and productive performance.
Cómo citar este artículo: MAYA-ORTEGA, CARLOS-ABEL; ÁNGEL-ISAZA, JAIMEANDRÉS; MARTÍNEZ-MORALES, BLANCA-CECILIA; PARRA-SUESCÚN, JAIMEEDUARDO. Aceite esencial de orégano (Lippia origanoides) mejora parámetros productivos y metabólitos sanguíneos en lechones. Biotecnología en el sector agropecuario y agroindustrial,v. 19, n. 2, 2021, p. 82-93. Doi: https://doi.org/10.18684/ bsaa.v19.n2.2021.1547

\section{PALABRAS CLAVE:}

PBS; PGPR; Suelos ácidos; Agricultura sustentable; Biotecnología; Biofertilizante; Bacterias endofítica; Fósforo; Café; Filogenética.

\section{KEYWORDS:}

PBS; PGPR; Acidic soils; Sustainable agriculture; Biotechnology; Biofertilizer; Endophytic bacteria; phosphorus; Coffee; Phylogenetics. 


\section{INTRODUCCIÓN}

El destete es considerado como una de las etapas más estresantes en la vida de los lechones, a tal punto que contribuye a disfunciones intestinales, particularmente durante las dos primeras semanas posteriores al destete (Tao et al., 2015). Dicha etapa, ha sido más crítica en los sistemas de producción modernos, en los cuales los lechones son destetados aproximadamente a los 21 días de edad; obteniendo como resultado alteraciones en la función de la barrera intestinal, disminuyendo la eficiencia en los procesos de digestión y afectando el crecimiento de los lechones (Goh et al., 2017).

Actualmente, los antibióticos han sido ampliamente utilizados en la alimentación animal bajo dosis sub terapéuticas, con el fin de contrarrestar el crecimiento de poblaciones bacterianas, las cuales pueden ser consideradas como patógenas a nivel intestinal, garantizando así una mejor salud del intestino y un mejor rendimiento productivo de los lechones durante el período posdestete (Kogut et al., 2017; Salim et al., 2018). Sin embargo, el uso de antibióticos como promotores de crecimiento ha traído consigo la aparición de cepas bacterianas resistentes a dichos compuestos, convirtiéndose en un riesgo para la salud pública (Liu et al., 2018; Yang et al., 2019).

Por dicha razón, en el año 2006, la Unión Europea prohibió el uso de antibióticos como promotores de crecimiento en la alimentación animal; mientras que otros países, como Estados Unidos, han venido realizando una regulación estricta en su uso (Hrnčár et al., 2016; Costa et al., 2017). Lo anterior, ha traído consigo consecuencias desfavorables en términos de productividad, bienestar y salud de los animales, principalmente a causa de la aparición de alteraciones fisiológicas estrechamente relacionadas con desequilibrios en el microbiota normal del intestino, lo cual se ve reflejado en un detrimento del rendimiento productivo (Costa et al., 2017). Dado este panorama, los investigadores se han dado a la tarea de buscar alternativas eficaces y biológicamente seguras que garanticen un buen rendimiento productivo mientras se protege la salud del consumidor (Sugiharto et al., 2016; Mehdi et al., 2018).

Colombia es considerado un país megadiverso, cuya flora alcanza más de 45.000 especies de plantas diferentes en su territorio, donde se encuentran a su vez, una amplia variedad de plantas aromáticas tanto nativas como exógenas, ambas de gran potencial económico debido a que pueden ser una fuente de nuevos compuestos bioactivos, cuyas potencialidades hasta el momento han sido poco estudiadas (Stashenko et al., 2010). Dentro de la amplia variedad de plantas nativas aromáticas, los investigadores han mostrado interés en algunas especies del género Lippia, las cuales hacer parte de la familia Verbenaceae Juss, cuyo material vegetal posee gran variedad de metabolitos secundarios bioactivos, dando lugar a diferentes clasificaciones de quimiotipos, debido a que sus diferentes componentes (extractos y aceites esenciales) poseen propiedades benéficas antifúngicas, antioxidantes y antibacterianas (Stashenko et al., 2014).

En los últimos años, se ha propuesto aprovechar las bondades de los aceites esenciales del género Lippia, al ser utilizados como aditivos nutricionales en la alimentación animal, ya que se ha evidenciado su capacidad para mejorar la estructura (morfología) y función intestinal, favorecer los procesos de digestión y absorción de nutrientes, modificar la composición de los ecosistemas microbianos intestinales, proteger los tejidos contra el estrés oxidativo, disminuir el gasto energético producto de los procesos de inflamación intestinal, y por ende, mejorar el rendimiento productivo de los animales (Londoño et al., 2016; Madrid et al., 2018; Mohiti and Moein, 2018; Betancourt et al., 2019; Patiño et al., 2019).

Por lo anterior, el objetivo del presente estudio fue evaluar el efecto de la suplementación con Lippia origanoides (familia Verbenaceae) sobre el rendimiento productivo y la concentración de metabolitos sanguíneos en lechones destetados a los 21 días. 


\section{MÉTODO}

\section{Consideraciones éticas}

Esta investigación está avalada por el Comité de Ética en la Experimentación Animal de la Universidad Nacional de Colombia, Sede Medellín (CEMED 012 del 04 de mayo de 2018). Todas las prácticas experimentales fueron llevadas siguiendo las guías propuestas por "The International Guiding Principles for Biomedical Research Involving Animals" (ICLAS, 2012).

\section{Localización}

El trabajo de campo se realizó en la Estación Agraria San Pablo, perteneciente a la Universidad Nacional de Colombia, sede Medellín, localizada en el departamento de Antioquia, municipio de Rionegro, en la vereda El Tablacito. La producción se localiza a una altitud aproximada de 2.100 m.s.n.m., zona de vida bosque muy húmedo Montano bajo (bmh-MB), con una temperatura entre 12 y $18^{\circ} \mathrm{C}$, una precipitación anual de $2.280 \mathrm{~mm}$ y una humedad relativa de $75 \%$.

\section{Animales}

Se utilizaron 72 lechones (machos) cruce terminal comercial (Duroc x Pietrain), destetados a los 21 días de edad con un peso aproximado de 5,6 $\pm 0,3 \mathrm{~kg}$. Todos los animales fueron alojados en grupos de 8 animales. El alimento y agua se ofreció a voluntad durante 30 días (etapa de precebo), y se llevó a cabo el registro del consumo de alimento de los diferentes grupos. Durante la lactancia no se les suministró alimento sólido y ningún tipo de antibiótico a los lechones.

\section{Manejo sanitario}

Para el recibimiento de los lechones se tuvieron en cuenta todos los protocolos de bioseguridad: lavado, limpieza y desinfección de instalaciones, cortinas, criadoras, comederos y bebederos. Adicionalmente, se realizó un estricto control de roedores e insectos con productos obtenidos en casas comerciales. Los lechones llegaron de lactancia con el plan de vacunación del sistema de producción establecido (Patiño et al., 2019).

\section{Instalaciones y equipos}

Los cerdos fueron alojados en corrales levantados (1,2 ×2,3 m), con piso de malla metálica. Los primeros 15 días del experimento se mantuvieron instaladas lechoneras de madera en los corrales, las cuales posteriormente se retiraron; además, se suministró calor por medio de gas producido por biodigestor para garantizar una temperatura promedio de $26^{\circ} \mathrm{C}$, controlándose la temperatura con cortinas (Patiño et al., 2019).

\section{Dietas}

Los animales fueron alimentados con una dieta comercial sin la adición de antibiótico, a la cual se le adicionó antibiótico (Bacitracina de zinc - APC) y aceite esencial de orégano Lippia origanoides, conformando cada una de las dietas así:

Dieta 1 o control (CONT): Alimento balanceado sin antimicrobiano.

Dieta 2 (ANT): D1 con la adición de antibiótico promotor de crecimiento (Bacitracina de Zinc) (350ppm).

Dieta 3 (AEO): D1 con la adición de aceite esencial de orégano (Lippia origanoides) (AEO) (150 ppm). 
Se elaboró una dieta experimental (Cuadro 1) que cubría los requerimientos mínimos nutricionales correspondiente a la etapa. El alimento utilizado en el estudio estuvo libre de antibióticos (excepto la dieta 2 (ANT), donde se utilizó Bacitracina de Zinc como APC, como se hace a nivel comercial). El AEO de Lippia origanoides (Kontra ${ }^{\circledR}$ Promitec S.A.S.) se adicionó a la pre mezcla a una concentración efectiva de 150 ppm para la dieta 3.

Cuadro 1. Composición nutricional de la dieta sin adición de antimicrobiano.

\begin{tabular}{|l|c|}
\hline \multicolumn{1}{|c|}{ Composición proximal } & Porcentaje \\
\hline Maíz extruido & $62,53 \%$ \\
\hline Torta de soya & $19,85 \%$ \\
\hline Concentrado proteico soya & $7,98 \%$ \\
\hline Aceite de palma & $4,50 \%$ \\
\hline Fosfato monodicálcico 21\% & $1,14 \%$ \\
\hline Carbonato de calcio & $0,98 \%$ \\
\hline Sal de mar & $0,36 \%$ \\
\hline Lisina HCl & $0,39 \%$ \\
\hline Metionina & $0,30 \%$ \\
\hline Treonina & $0,15 \%$ \\
\hline Triptófano & $0,05 \%$ \\
\hline
\end{tabular}

\section{Variables productivas}

La colección de datos productivos para calcular los parámetros zootécnicos fue realizada los días 1 y 30 posdestete. Se registró la cantidad de alimento ofrecido y rechazado para calcular consumo total y peso corporal; además, se calculó la conversión alimenticia para estas mismas fechas (Londoño et al., 2016).

Ganancia Diaria de Peso (GDP). Explica cuánto ganan los animales diariamente (Ec 1).

$$
G A P=\frac{\text { Peso final }- \text { Peso inicial }}{\text { Edad }(\text { días })}
$$

Índice de Conversión Alimenticia (CA). Permite cuantificar cuántos kilogramos de alimento necesita un animal para producir un kilogramo de carne (Ec.2).

$$
C A=\frac{\text { Alimento consumido }}{\text { Peso ganado }}
$$

\section{Toma de muestras}

Se colectaron $10 \mathrm{~mL}$ de sangre de la vena yugular en un tubo sin anticoagulante los días 1,15 y 30 posdestete. Las muestras fueron centrifugadas de inmediato por medio de una centrifuga 800-1 (CDEK®) a 4000 rpm por 5 minutos, para luego ser almacenadas en frio y transportadas el mismo día al Laboratorio para su análisis. Los metabolitos evaluados fueron: creatinina, alanino aminotransferasa (ALT), fosfatasa alcalina, glucosa, colesterol, triglicéridos, fósforo y calcio. La cuantificación de los analitos en suero se realizó mediante los estuches comerciales Slides VITROS ${ }^{\circ}$ (Ortho Clinical Diagnostics, EEUU) específicos para cada analito, siguiendo las instruc- 
ciones del fabricante. Para la medición de las densidades ópticas se utilizó un analizador bioquímico VITROS $®$ DT- 60II (Ortho Clinical Diagnostics, EEUU) (Londoño and Parra, 2015).

\section{Diseño experimental y análisis estadístico}

Para el análisis de la información se realizó un modelo de medidas repetidas en el tiempo bajo un diseño completamente al azar con tres tratamientos (dieta) y tres repeticiones (8 lechones por repetición). Cada uno de los animales fue asignado aleatoriamente a una de las cuatro dietas. El análisis estadístico se realizó según el procedimiento Proc Mixed del SAS ${ }^{\text {. El }}$ procedimiento de comparaciones múltiples se realizó mediante una prueba de Tukey.

\section{RESULTADOS}

En general, durante el periodo de experimentación los lechones presentaron un buen estado de salud, sin la aparición de algún signo clínico adverso que implicara su retiro y/o sacrificio antes de culminar el periodo experimental.

En el cuadro 2 se presenta un resumen del rendimiento zootécnico de los lechones adicionados con las diferentes dietas. Se puede observar que la ganancia diaria de peso (GDP) incrementó de manera significativa $(p<0,05)$ en aquellos lechones que recibieron aceite esencial de orégano (AEO) en su dieta en comparación con aquellos lechones que recibieron Bacitracina de zinc (ANT) como promotor de crecimiento. Por su parte, se observó una disminución significativa $(p<0,05)$ en la tasa de conversión alimenticia promedio (CAP) a lo largo del periodo experimental mediante el uso de AEO en la dieta frente los resultados obtenidos con los tratamientos control (CONT) y ANT. Sin embargo, el consumo de alimento no presentó una variación significativa $(p>0,05)$ entre las diferentes dietas. Dada una disminución significativa en la tasa de conversión alimenticia en los echones adicionados con AEO y un consumo estadísticamente igual entre los diferentes tratamientos, se puede afirmar que la adición de aceite esencial de orégano (Lippia origanoides) mejoró la eficiencia en el aprovechamiento del alimento.

Cuadro 2. Influencia en la adición de aceite esencial de orégano (Lippia origanoides) sobre el rendimiento productivo de lechones destetados a los 21 días.

\begin{tabular}{|l|c|c|c|c|}
\hline \multicolumn{1}{|c|}{ Parámetro } & \multicolumn{3}{c|}{ Dieta } & Valor $\mathbf{p}$ \\
\hline & CONT & ANT & AEO & \\
\hline Conversión alimenticia & $1,9^{\mathrm{A}}$ & $1,815^{\mathrm{B}}$ & $1,58^{\mathrm{C}}$ & $4,93 \mathrm{e}-12$ \\
\hline Ganancia de peso $(\mathrm{g})$ & $361^{\mathrm{C}}$ & $383^{\mathrm{B}}$ & $444^{\mathrm{A}}$ & $1,13 \mathrm{e}-13$ \\
\hline Consumo (Kg) & $20,6^{\mathrm{A}}$ & $20,8^{\mathrm{A}}$ & $21,0^{\mathrm{A}}$ & 0,72 \\
\hline
\end{tabular}

(CONT): dieta sin la adición de antibiótico, probiótico y AEO. (ANT): dieta control más la adición de antibiótico (Bacitracina de zinc 350 ppm). (AEO): dieta control más la adición de 150 ppm de aceite esencial de orégano (AEO-Lippia origanoides).

$A, B, C$ Dentro de una misma fila, medias con un superíndice común (por variable en estudio) no difieren estadísticamente $(p>0,05)$

Los valores promedio en las concentraciones séricas de creatinina y de las enzimas alanina aminotransferasa y fosfatasa alcalina se presentan en el cuadro 3. La adición de AEO disminuyó de manera significativa $(p<0,05)$ las concentraciones de alanina aminotransferasa y creatinina en comparación a los demás tratamientos; mientras se evidenció un aumento significativo $(p<0,05)$ en la concentración sérica de fosfatasa alcalina en aquellos lechones que recibieron AEO.

En el cuadro 4 se presentan los resultados para la concentración séricas de los metabolitos glucosa, triglicéridos y colesterol, los cuales están estrechamente relacionados con el metabolismo energético en monogástricos. Las concentraciones séricas de glucosa aumentaron de manera significativa $(p<0,05)$ en aquellos lechones que recibieron AEO en su alimento; además, el uso de AEO disminuyó de manera significativa las concentraciones sanguíneas de colesterol y triglicéridos libres en los lechones. Sin embargo, los lechones que recibieron antibiótico 
como promotor de crecimiento no presentaron una variación significativa en las concentraciones de colesterol sérico en comparación con el tratamiento control al final del día 30 posterior al destete.

Cuadro 3. Efecto del uso de aceite esencial de orégano (Lippia origanoides) sobre la concentración sérica de alanina amino-transferasa, creatinina y fosfatasa en lechones destetados a los 21 días.

\begin{tabular}{|c|c|c|c|c|c|}
\hline Parámetro & Edad & CONT & ANT & AEO & Valor $p$ \\
\hline \multirow{3}{*}{$\begin{array}{l}\text { Alanina amino-transfe- } \\
\text { rasa (UI/L) }\end{array}$} & 0 días & $63,00^{\mathrm{A}}$ & $62,76^{\mathrm{A}}$ & $62,79^{A}$ & 0,984 \\
\hline & 15 días & $56,50^{A}$ & $50,00^{\mathrm{B}}$ & $48,50^{B}$ & 6,18 e -07 \\
\hline & 30 días & $49,6^{\mathrm{A}}$ & $44,00^{B}$ & $42,70^{B}$ & 5,93 e - 07 \\
\hline \multirow{3}{*}{ Creatinina (mg/dL) } & 0 días & $1,25^{\mathrm{A}}$ & $1,25^{\mathrm{A}}$ & $1,25^{\mathrm{A}}$ & 0,989 \\
\hline & 15 días & $1,12^{\mathrm{A}}$ & $1,00^{\mathrm{B}}$ & $0,86^{c}$ & 3,96 e -09 \\
\hline & 30 días & $0,99^{A}$ & $0,88^{\mathrm{B}}$ & $0,76^{c}$ & 5,58 e -07 \\
\hline \multirow{3}{*}{ Fosfatasa (mg/dL) } & 0 días & $201,90^{A}$ & $202,15^{\mathrm{A}}$ & $201,87^{A}$ & 0,696 \\
\hline & 15 días & $229,4^{C}$ & $232,4^{\mathrm{B}}$ & $266,5^{\mathrm{A}}$ & $2 e-16$ \\
\hline & 30 días & $256,9^{c}$ & $260,3^{\mathrm{B}}$ & $298,5^{\mathrm{A}}$ & $2 e-16$ \\
\hline
\end{tabular}

(CONT): dieta sin la adición de antibiótico, probiótico y AEO. (ANT): dieta control más la adición de antibiótico (Bacitracina de zinc $350 \mathrm{ppm}$ ). (AEO): dieta control más la adición de 150 ppm de aceite esencial de orégano (AEO-Lippia origanoides). ${ }^{\mathrm{A}, \mathrm{B}, \mathrm{C}} \mathrm{C}$ Dentro de una misma fila, medias con un superíndice común (por variable en estudio) no difieren estadísticamente $(p>0,05)$.

Cuadro 4. Influencia en la adición de aceite esencial de orégano (Lippia Origanoides) sobre la concentración sérica de glucosa, colesterol y triglicéridos en lechones destetados a los 21 días.

\begin{tabular}{|l|c|c|c|c|c|}
\hline \multicolumn{1}{|c|}{ Parámetro } & Edad & CONT & ANT & AEO & Valor $\mathbf{p}$ \\
\hline \multirow{3}{*}{ Glucosa $(\mathrm{mg} / \mathrm{dL})$} & 0 días & $101,8^{\mathrm{A}}$ & $101,7^{\mathrm{A}}$ & $102,12^{\mathrm{A}}$ & 0,662 \\
\cline { 2 - 6 } & 15 días & $115,7^{\mathrm{C}}$ & $121,2^{\mathrm{B}}$ & $136,0^{\mathrm{A}}$ & $2 \mathrm{e}-16$ \\
\cline { 2 - 6 } & 30 días & $129,6^{\mathrm{C}}$ & $135,7^{\mathrm{B}}$ & $152,2^{\mathrm{A}}$ & $2 \mathrm{e}-16$ \\
\hline \multirow{3}{*}{ Colesterol (mg/dL) } & 0 días & $119,02^{\mathrm{A}}$ & $118,93^{\mathrm{A}}$ & $119,02^{\mathrm{A}}$ & 0,619 \\
\cline { 2 - 6 } & 15 días & $106,2^{\mathrm{A}}$ & $105,0^{\mathrm{A}}$ & $99,5^{\mathrm{B}}$ & $5,98 \mathrm{e}-09$ \\
\cline { 2 - 6 } & 30 días & $93,5^{\mathrm{A}}$ & $92,4^{\mathrm{A}}$ & $87,6^{\mathrm{B}}$ & $2,96 \mathrm{e}-07$ \\
\hline \multirow{3}{*}{ Triglicéridos $(\mathrm{mg} / \mathrm{dL})$} & 0 días & $106,6^{\mathrm{A}}$ & $106,21^{\mathrm{A}}$ & $105,72^{\mathrm{A}}$ & 0,482 \\
\cline { 2 - 6 } & 15 días & $95,2^{\mathrm{A}}$ & $93,6^{\mathrm{A}}$ & $84,05^{\mathrm{B}}$ & $1,28 \mathrm{e}-11$ \\
\cline { 2 - 6 } & 30 días & $83,8^{\mathrm{A}}$ & $82,4^{\mathrm{B}}$ & $75,63^{\mathrm{C}}$ & $3,45 \mathrm{e}-12$ \\
\hline
\end{tabular}

(CONT): dieta sin la adición de antibiótico, probiótico y AEO. (ANT): dieta control más la adición de antibiótico (Bacitracina de zinc $350 \mathrm{ppm}$ ). (AEO): dieta control más la adición de 150 ppm de aceite esencial de orégano (AEO-Lippia origanoides). ${ }^{A, B, C}$ Dentro de una misma fila, medias con un superíndice común (por variable en estudio) no difieren estadísticamente $(p>0,05)$.

Un resumen de las concentraciones séricas de calcio y fósforo son presentadas en el cuadro 5 , se puede observar, que el uso de aceite esencial de orégano incrementó de manera significativa $(p<0,05)$ las concentraciones séricas de calcio y fósforo en los lechones.

Cuadro 5. Efecto del uso de aceite esencial de orégano (Lippia Origanoides) sobre la concentración sérica de fósforo y calcio en lechones destetados a los 21 días.

\begin{tabular}{|c|c|c|c|c|c|}
\hline Parámetro & Edad & CONT & ANT & AEO & Valor $\mathrm{p}$ \\
\hline \multirow{3}{*}{ Fósforo (mg/dL) } & 0 días & $6,42^{\mathrm{A}}$ & $6,415^{\mathrm{A}}$ & $6,415^{\mathrm{A}}$ & 0,977 \\
\hline & 15 días & $7,30^{\circ}$ & $7,40^{\mathrm{B}}$ & $7,60^{A}$ & 1,02 e -08 \\
\hline & 30 días & $8,18^{c}$ & $8,29^{\mathrm{B}}$ & $8,51^{\mathrm{A}}$ & $1,35 \mathrm{e}-10$ \\
\hline \multirow{3}{*}{ Calcio (mg/dL) } & 0 días & $9,51^{\mathrm{A}}$ & $9,50^{\mathrm{A}}$ & $9,44^{\mathrm{A}}$ & 0,117 \\
\hline & 15 días & $10,60^{c}$ & $10,80^{\mathrm{B}}$ & $10,91^{\mathrm{A}}$ & $1,21 \mathrm{e}-06$ \\
\hline & 30 días & $11,90^{c}$ & $12,10^{\mathrm{B}}$ & $12,20^{A}$ & 3,82 e - 07 \\
\hline
\end{tabular}


En general, se observa que la edad posterior al destete es un factor que influye en el metabolismo de los cerdos, pues acorde avanza el tiempo después del destete se disminuyen las concentraciones de algunas enzimas y metabolitos como la alanina aminotransferasa, creatinina, colesterol y triglicéridos, mientras se incrementan las concentraciones de fosfatasa alcalina, glucosa, calcio y fosforo.

El destete es un evento que trae consigo diferentes retos nutricionales que están estrechamente relacionados con el desarrollo intestinal y la eficiencia productiva en la vida del animal (Omonijo et al., 2018; Salim et al., 2018). Así, desequilibrios en la salud del intestino comprometen los procesos de digestión-absorción y, por ende, la disponibilidad de energía y nutrientes presentes en el alimento para garantizar el mantenimiento y el desarrollo de los diferentes tejidos en los (Wang et al., 2018; Gao et al., 2019; Modina et al., 2019).

Son diversos los mecanismos mediante los cuales, los aceites esenciales permiten mejorar el rendimiento productivo en los animales de granja. Estudios realizados en cerdos y aves de corral han evidenciado que el uso de aceite esencial de orégano, específicamente de Lippia origanoides en la alimentación animal ha permitido obtener una mayor tasa de crecimiento, al mejorar los procesos de digestión y absorción de los nutrientes, disminuir los procesos inflamatorios intestinales, y garantizar la eubiosis en los ecosistemas microbianos presentes a lo largo del tracto gastrointestinal de los animales (Zou et al., 2016; Zhai et al., 2018). Mejoras significativas en el rendimiento productivo de cerdos y aves que recibieron AEO como alternativa al uso de antibióticos promotores del crecimiento han sido reportados en diversas investigaciones (Peng et al., 2016; Cheng et al., 2018; Liu et al., 2018; Madrid et al., 2018). Una mayor digestibilidad de nutrientes del alimento, un mayor desarrollo de las vellosidades, el incremento en el área superficial del epitelio intestinal, y la disminución en el gasto catabólico por una disminución en la inflamación intestinal y regeneración de la morfología del intestino, son los principales factores asociados a un mayor rendimiento productivo bajo el uso de aceite esencial de orégano (Van Der Aar et al., 2017; Cheng et al., 2018; Omonijo et al., 2018).

Enzimas como la alanina aminotransferasa (ALT) son cuantificadas a nivel sérico y utilizadas como biomarcadores para evaluar la funcionalidad e integridad del hígado y descartar la presencia de lesiones o alteraciones causadas por desórdenes metabólicos, toxinas, infecciones o inflamaciones crónicas; mientras la concentración de creatinina es un indicativo en la funcionalidad del riñón (Adeyemi et al., 2015; Hasan et al., 2018). Así, una disminución en las concentraciones séricas de ALT y creatinina está relacionada con un buena funcionalidad e integridad del hígado y riñón respectivamente (Gwaltney, 2016).

Por su parte, la fosfatasa alcalina es una enzima estrechamente relacionada con la utilización del fósforo y el desarrollo óseo, por tanto, valores altos están relacionados con una alta disponibilidad de fósforo y con el desarrollo óseo de animales en crecimiento (Adeyemi et al., 2015). De acuerdo con los resultados encontrados en el presente estudio, ha sido reportado que el uso de AEO en la dieta disminuye las concentraciones séricas de ALT y creatinina, e incrementa la presencia de fosfatasa alcalina de manera significativa con respecto al uso de antibióticos promotores de crecimiento (Madrid et al., 2018; Gao et al., 2020).

La glucosa es un metabolito indispensable en los procesos fisiológicos de animales monogástricos, ya que representa una fuente de energía esencial para el mantenimiento y desarrollo de tejidos (Thongsong et al., 2019). Sin embargo, factores asociados al desarrollo de las vellosidades intestinales y la expresión de transportadores intestinales de glucosa afectan la absorción de dicho nutriente, y por tanto su disponibilidad a nivel sanguíneo, afectando el crecimiento de los animales (Gao et al., 2020; Karadağoğlu et al., 2020). Estudios realizados en aves y cerdos respaldan los resultados presentados en el cuadro 4, pues se ha reportado que el uso de AEO en la dieta incrementa las concentraciones séricas de glucosa en comparación al uso de antibióticos promotores del crecimiento (Patiño et al., 2019).

La disminución en los niveles séricos de colesterol y triglicéridos libres está directamente relacionada con una menor tasa de problemas cardiacos (Madrid et al., 2018) y una menor deposición de grasa muscular en los animales (Karadağoğlu et al., 2020), lo cual se verá reflejado en una menor tasa de mortalidad en granja y una mejor aceptación del producto final por parte de los consumidores. Acorde a los resultados encontrados en la 
presente investigación, se ha encontrado una disminución en los valores séricos de colesterol y triglicéridos de lechones y pollos de engorde que recibieron AEO en la dieta (Patiño et al., 2019). Dicha disminución ha sido asociada a un incremento en la concentración sérica de lipoproteínas de alta densidad (HDL) que facilitan el transporte de colesterol y triglicéridos libres nuevamente al hígado (Li et al., 2017), y una consecuente reducción en la concentración de lipoproteínas de baja densidad (LDL) inducida bajo el uso de AEO (Madrid et al., 2018). Además, ha sido propuesto que los fenoles presentes en los aceites esenciales tienen la capacidad de regular la homeostasis del colesterol mediante un aumento en la expresión de la enzima colesterol 7 alfa-hidrolasa implicada en la síntesis de sales biliares, y la inhibición en la actividad de la 3-hidroximetil glutaril-COA cuya función es limitante en la síntesis de colesterol (Karadağoğlu et al., 2020).

El incremento en la altura y ancho de las vellosidades intestinales mediante el uso de AEO, permite incrementar el área de contacto entre el epitelio del intestino y el contenido intestinal, lo cual aumenta la eficiencia en la absorción de minerales (Wang et al., 2019; Li et al., 2020; Wang et al., 2020) favoreciendo una mayor disponibilidad de calcio y fósforo a nivel sérico; minerales indispensables en el desarrollo y mantenimiento de la estructura ósea de los animales, y en el caso específico del fósforo, en el metabolismo energético como componente fundamental del ATP (Li et al., 2017; Singh et al., 2018). Estudios realizados por Patiño et al., (2019) reportan un aumento en las concentraciones séricas de calcio y fósforo durante el periodo post-destete en lechones que recibieron AEO en su dieta; resultados similares fueron reportados por Madrid Garcés et al., (2019) en pollos de engorde.

\section{CONCLUSIONES}

El uso de aceite esencial de orégano (Lippia origanoides) constituye una alternativa para la sustitución parcial en el uso de antibióticos promotores del crecimiento en la alimentación de lechones durante el período posdestete, puesto que mejora el perfil lipídico a nivel sérico, la concentración de fosfatasa alcalina y la disponibilidad de nutrientes como la glucosa, calcio y fósforo, indispensables para la salud y el correcto desempeño del animal; además, de disminuir las concentraciones séricas de ALT y creatinina relacionadas con el buen funcionamiento e integridad del hígado y riñón respectivamente.

\section{REFERENCIAS}

ADEYEMI, OLAOLUWA; ADEBAWO, OLUGBENGA; AFOLAYAN, JOSEPH; ONAJOBI, FUNMILAYO; OSILESI, ODUTOLA; OYEDEMI, SUNDAY. Alkaline phosphatase (ALP), aspartate aminotransferase (AST) and alanine aminotransferase (ALT) Activities in selected tissues of rats fed on processed atlantic horse mackerel (Trachurus trachurus). Advances in Bioscience and Biotechnology, v. 6, n. 3, 2015, p. 139-152. 10.4236/abb.2015.63014.

BETANCOURT, LILIANA; AFANADOR, GERMÁN; HUME, MICHAEL; NISBET, DAVID; RODRÍGUEZ, FERNANDO; SOHAIL, MUHAMAD. Umar. Effects of Colombian oregano essential oil (Lippia origanoides Kunth) and Eimeria species on broiler production and cecal microbiota. Poultry science, v. 98, n. 10, 2019, p. 4777-4786. https://doi.org/10.3382/ps/pez193

CHENG, CHUANSHANG; JIANG, SIWEN; PENG, JIAN; WANG, CHAO; XIA, MAO; ZANG, XIAMING. Supplementing oregano essential oil in a reduced-protein diet improves growth performance and nutrient digestibility by modulating intestinal bacteria, intestinal morphology, and antioxidative capacity of growing-finishing pigs. Animals, v. 8, n. 9, 2018, p. 159. https://doi.org/10.3390/ani8090159.

COSTA, MARCIO; ALFIERI, AMAURI; BESSEGATO, JOSE; FILHO, JOÃO; OBA, ALEXANDRE; WEESE, SCOTT. Different antibiotic growth promoters induce specific changes in the cecal microbiota membership of broiler chicken. PLoS ONE, v. 12, n. 2, 2017, p. 1-14.

https://doi.org/10.1371/journal.pone.0171642 
HASAN, KAZI; HAQUE, M.D. ANWARUL; TAMANNA, NASRIN. Biochemical and histopathological profiling of Wistar rat treated with Brassica napus as a supplementary feed. Food science and human wellness, v. 7, n. 1, 2018, p. 77-82. https://doi.org/10.1016/j.fshw.2017.12.002.

HRNČÁR, CYRIL; ARPÁŠOVÁ, HENRIETA; BUJKO, JOZEF; FIK, MARTIN; GAŠPAROVIČ, MARTIN; PISTOVÁ, VERONIKA; WEIS, JÁN. Effect of three-strain probiotic on productive performance and carcass characteristics of broiler chickens. Scientific Papers: Animal Science and Biotechnologies, v. 49, n. 2, 2016, p. 149-154.

KARADAĞOĞLU, ÖZLEM; ÖLMEZ, MÜKREMIN; ÖZSOY, BÜLENT; ŞAHIN, TARKAN; YAKAN, AKIN. Changes in serum biochemical and lipid profile, and fatty acid composition of breast meat of broiler chickens fed supplemental grape seed extract. Turkish Journal of Veterinary and Animal Sciences, v. 44, n. 2, 2020, p. 182-190. https://doi.org/10.3906/vet-1906-37.

KOGUT, MICHAEL; BROOM, LEON; YIN, XIAONAN; YUAN, JIANMIN. Gut health in poultry. CAB Reviews: Perspectives in agriculture, veterinary science, nutrition and natural resources, v. 12, n. 031, 2017, p. 1-7. https://doi.org/10.1079/PAVSNNR201712031

GAO, XIAOQIAN; CHEN, DAIWEN; HE, JUN; HUANG, ZHIQING; LUO, JUNQIU; LUO, YUHENG; MAO, XIANGBING; YU, BING; YU, JIE; ZHENG, PING. Effects of dietary starch structure on growth performance, serum glucose-insulin response, and intestinal health in weaned piglets. Animals, v. 10, n. 3, 2020, p. 1-16. https://doi.org/10.3390/ani10030543.

GAO, JING; LI, TIEJUN; XU, KANG; YIN, JIE; YIN, YULONG. What Is the Impact of Diet on Nutritional Diarrhea Associated with Gut Microbiota in Weaning Piglets: A System Review. BioMed Research International, v. 2019, n. 0, 2019. https://doi.org/10.1155/2019/6916189

GOH, T.W.; KIM, B.O.; DO, S.H.; KIM, Y.Y.; YOO, D.H. 202 Various levels of milk by-products in weaning pig diet on growth performance, blood profiles, intestinal morphology, muscle fiber diameter, and diarrhea incidence in weaning pigs. Journal of Animal Science, v. 95, n. 2, 2017, p. 96-97. https://doi.org/10.2527/asasmw.2017.12.202

GWALTNEY-BRANT, SHARON. Nutraceuticals in hepatic diseases. En Nutraceuticals. Salt Lake City (United States Of America): Academic Press, 2016. p. 87-99. https://doi.org/10.1016/B978-0-12-802147-7.00007-3.

LIU, YANHONG; ABELILLA, JERUBELLA; CASAS, GLORIA; ESPINOSA, CHARMAINE; JAWORSKI, NEIL; KWON, WOONG; LAGOS, VANESSA; LEE, SU; MATHAI, JHON; NAVARRO, DIEGO; STEIN, HANS. Non-antibiotic feed additives in diets for pigs: a review. Animal nutrition, v. 4, n. 2, 2018, p. 113-125. https://doi.org/10.1016/j.aninu.2018.01.007

LI, X.; BRYDEN, W.; ZHANG, D. Calcium and phosphorus metabolism and nutrition of poultry: Are current diets formulated in excess? Animal Production Science, v. 57, n. 11, 2017, p. 2304-2310. https://doi.org/10.1071/AN17389

LI, HUIFENG; CHENG, JIN; LUO, RONG; YUAN, YITONG;ZHU,ZHIWEI. Age-related intestinal monosaccharides transporters expression and villus surface area increase in broiler and layer chickens. Journal of Animal Physiology and Animal Nutrition, v. 104, n. 1, 2020, p. 144-155. https://doi.org/10.1111/jpn.13211.

LONDOÑO, SANTIAGO; LALLĖS, JEAN-PAUL; PARRA, JAIME-EDUARDO. Effect of probiotic strain addition on digestive organ growth and nutrient digestibility in growing pigs. Revista Facultad Nacional de Agronomía Medellín, v. 69, n. 2, 2016, p. 7911-7918. http://dx.doi.org/10.15446/rfna.v69n2.59136.

LONDOÑO, SANTIAGO; PARRA, JAIME-EDUARDO. Efecto de la adición de cepas probióticas sobre metabolitos sanguíneos en cerdos en crecimiento. Biotecnología en el Sector Agropecuario y Agroindustrial, v. 13, n. 2, 2015, p. 49-56. https://doi.org/10.18684/BSAA(13)49-56 
MADRID, TOMÁS-ANTONIO; LÓPEZ, ALBEIRO; PARRA, JAIME-EDUARDO. Efecto del aceite esencial de orégano (Lippia origanoides) sobre metabolitos sanguíneos en pollos de engorde. Revista de Medicina Veterinaria, v. 37, n. 1, 2018, p. 25-33. https://doi.org/10.19052/mv.vol1.iss37.3.

MADRID, TOMÁS-ANTONIO; LÓPEZ, ALBEIRO; PARRA, JAIME-EDUARDO. La ingesta de aceite esencial de orégano (Lippia origanoides) mejora la morfología intestinal en Broilers. Archivos de zootecnia, v. 67, n. 260, 2018, p. 470-476. https://doi.org/10.21071/az.v0i0.3876.

MEHDI, YOUCEF; CHORFI, YOUNES; GAUCHER, MARIE-LOU; LÉTOURNEAU-MONTMINY, MARIE- PIERRE; ROUISSI, TAREK; SURESH, GAYATRI. Use of antibiotics in broiler production: Global impacts and alternatives. Animal nutrition, v. 4, n. 2, 2018, p. 170-178. https://doi.org/10.1016/j.aninu.2018.03.002

MODINA, SILVIA-CLOTILDE; CORINO, CARLO; GIANCAMILLO, ALESSIA; POLITO, UMBERTO; ROSSI, RAFAELLA. Nutritional Regulation of Gut Barrier Integrity in Weaning Piglets. Animals, v. 9, n. 12, 2019, p. 1-16. https://doi.org/10.3390/ani9121045

MOHITI, MAZIAR; GHANAATPARAST, MOEIN. Comparing the effects of a combined phytogenic feed additive with an individual essential oil of oregano on intestinal morphology and microflora in broilers. Journal of Applied Animal Research, v. 46, n. 1, 2018, p. 184-189. https://doi.org/10.1080/09712119.2017.1284074.

NIU, JUN; NIE, CUN-XI; WEI, LIAN-QING; ZHANG, JUN; ZHANG, WWEN-HU. Effect of Fermented Cottonseed Meal on the Lipid-Related Indices and Serum Metabolic Profiles in Broiler Chickens. Animals, v. 9, n. 1, 2019 , p. 930. https://doi.org/10.3390/ani9110930

OMONIJO, FAITH; GONG, JOSHUA; LAHAYE, LUDOVIC; NI, LIJU; WANG, QI; YANG, CHENGBO. Essential oils as alternatives to antibiotics in swine production. Animal Nutrition, v. 4, n. 2, 2018, p. 126-136. https://doi.org/10.1016/j.aninu.2017.09.001

PATIÑO, FELIPE; HERRERA, VICTOR-HUGO; LOPEZ, DANIELA; PARRA, JAIME-EDUARDO. Blood metabolites and zootechnical parameters in piglets weaned at two ages and with the addition of antimicrobials in the feed. Revista de Investigaciones Veterinarias Del Peru, v. 30, n. 2, 2019, p. 612-623. https://doi.org/10.15381/rivep.v30i2.14887

PENG, Q.; DUAN, Z.Y.; LI, J.D.; WU, Y.P. Effects of dietary supplementation with oregano essential oil on growth performance, carcass traits and jejunal morphology in broiler chickens. Animal Feed Science and Technology, v. 214, n. 1, 2016, p. 148-153. https://doi.org/10.1016/j.anifeedsci.2016.02.010.

SALIM, HOSSAN M.D.; HAQUE-BEG, M.D. ANWARUL; HUQUE, KHAN-SHAHIDUL; KAMARUDDIN, KAZI M. Global restriction of using antibiotic growth promoters and alternative strategies in poultry production. Science progress, v. 101, n. 1, 2018, p. 52-75. https://doi.org/10.3184/003685018X15173975498947

SINGH, J.; HUNDAL, J.S.; SETHI, A.P.; SHARMA, A.; SINGH, P.; SINGH, U. Phosphorus Nutrition in Dairy Animals: A Review. International Journal of Current Microbiology and Applied Science, v. 7, n. 4, 2018, p. 35183530. https://doi.org/10.20546/ijcmas.2018.704.397

STASHENKO, ELENA; ARIAS, GINNA; CALA, MÓNICA; DURÁN, CAMILO; MARTÍNEZ, JAIRO; SALGAR, WILLIAM. Lippia origanoides chemotype differentiation based on essential oil GC-MS and principal component analysis. Journal of Separation Science, v. 33, n. 1, 2010, p. 93-103. https://doi.org/10.1002/jssc.200900452

STASHENKO, ELENA; CABALLERO, DEYANIRA; CORDOBA, YURI; DURÁN, DIEGO-CAMILO; MARTÍNEZ, JAIRO-RENÉ. Estudio comparativo de la composición química y la actividad antioxidante de los aceites esenciales de algunas plantas del género Lippia (Verbenaceae) cultivadas en Colombia. Revista de La Academia Colombiana de Ciencias Exactas, Físicas y Naturales, v. 38, n. 0, 2014, p. 89-105. https://doi.org/10.18257/raccefyn.156 
SUGIHARTO, SUGIHARTO. Role of nutraceuticals in gut health and growth performance of poultry. Journal of the Saudi Society of Agricultural Sciences, v. 15, n. 2, 2016, p. 99-111. https://doi.org/10.1016/j.jssas.2014.06.001

TAO, XIN; XU, ZIWEI; MEN, XIAOMIN. Transient changes of enzyme activities and expression of stress proteins in the small intestine of piglets after weaning. Archives of Animal Nutrition, v. 69, n. 3, 2015, p. 201-211. https://doi.org/10.1080/1745039X.2015.1034828

THONGSONG, B.; KALANDAKANOND-THONGSONG, S.; WIYAPORN, M. Blood glucose, amino acid profiles and nutrient transporter gene expressions in the small intestine of low and normal birthweight piglets during the early suckling period. The Veterinary Journal, v. 247, n. 1, 2019, p. 1-7. https://doi.org/10.1016/j.tvjl.2019.02.006.

YANG, HONG; CLARKE, JIHONG-LIU; CHEN, XUNJI; LIU, DI; PARUCH, LISA; SKOMEDAL, HANNE; VAN-EERDE, ANDRE; WANG, YANLIANG. Antibiotic application and resistance in swine production in China: current situation and future perspectives. Frontiers in veterinary science, v. 6, n. 136, 2019, p. 1-8. https://doi.org/10.3389/fvets.2019.00136

VAN DER AAR, P.; MOLIST, F.; VAN DER KLIS, J. The central role of intestinal health on the effect of feed additives on feed intake in swine and poultry. Animal feed science and technology, v. 233, n. 1, 2017, p. 64-75. https://doi.org/10.1016/j.anifeedsci.2016.07.019.

WANG, LIXIA; DING, XUEQIN; LI, JIANZHONG; LI, YALI; XIONG, XIA; YANG, HUANSHENG; YIN, YULONG; ZHU, FAN. Effects of dietary supplementation with epidermal growth factor on nutrient digestibility, intestinal development and expression of nutrient transporters in early-weaned piglets. Journal of animal physiology and animal nutrition, v. 103, n. 2, 2019, p. 618-625. https://doi.org/10.1111/jpn.13059

WANG, MIN;DING,XUEQUIN;HUANG,PENGFEI;LI,JIANZHONG;LI,YALI;XIONG,XIA;YANG, HUANSHENG; YIN, YULONG; ZHU, FAN. The relationship between villous height and growth performance, small intestinal mucosal enzymes activities and nutrient transporters expression in weaned piglets. Journal of Animal Physiology and Animal Nutrition, v. 104, n. 2, 2020, p. 606-615. https://doi.org/10.1111/jpn.13299

WANG, YANBO; HUANG, BAJORA; SUN, SHENG; XIANG, HONGYU; XIE, QIUHONG; XU, YUN; ZANG, SHUMIN; ZANG, YING. Probiotics-fermented Massa Medicata Fermentata ameliorates weaning stress in piglets related to improving intestinal homeostasis. Applied microbiology and biotechnology, v. 102, n. 24, 2018, p. 10713-10727. https://doi.org/10.1007/s00253-018-9438-y

ZHAI, HENGXIAO; KLUENTER, ANNA-MARIA; LIU, HONG; WANG, SHIKUI; WU, JINLONG. Potential of essential oils for poultry and pigs. Animal nutrition, v. 4, n. 2, 2018, p. 179-186. https://doi.org/10.1016/j.aninu.2018.01.005

ZOU, YI; PENG, JIAN; WANG, JUN; WEI, HONG; XIANG, QUANHANG. Oregano essential oil improves intestinal morphology and expression of tight junction proteins associated with modulation of selected intestinal bacteria and immune status in a pig model. BioMed Research International, v. 2016, n. 1, 2016, p. 1-11.

https://doi.org/10.1155/2016/5436738 